\title{
APLICAÇÃO DE TÉCNICAS DE OTIMIZAÇÃO NA DISTRIBUIÇÃO DE CARVÕES EM DOSADORAS NA ARCELORMITTAL AÇOS PLANOS AMÉRICA DO SUL*
}

\author{
Pedro Lucas Freitas ${ }^{1}$ \\ Clebson Joel Mendes de Oliveira ${ }^{2}$ \\ Ubiratan Lauterjung dos Reis ${ }^{3}$
}

\begin{abstract}
Resumo
A elaboração de planos de mistura é feita em uma coqueria para uniformizar a qualidade das misturas. Um problema recorrente é a distribuição destes carvões nas dosadoras para serem misturados. A distribuição, no plano, destes carvões nas dosadoras é feita de forma manual, levando em consideração apenas o conhecimento do operador. A proposta é apresentar uma modelagem matemática de programação linear para resolver o problema da distribuição de carvões em dosadoras, segundo restrições de utilização das dosadoras dentro da faixa ótima, composição da mistura e uniformização das correias transportadoras, para reduzir sobrecarga nas linhas de dosadoras, com a finalidade de execução de um plano de mistura de carvões. Foram utilizados métodos de pesquisa operacional utilizando o algoritmo Simplex.
\end{abstract}

Palavras-chave: Programação linear; Pesquisa operacional; Mistura de carvões; Simplex.

\section{APPLICATION OF OPTIMIZATION TECHNICS IN COALS DISTRIBUTION IN CONSTANT FEEDWEIGHT OF ARCELORMITTAL FLAT CARBON SOUTH AMERICA}

\section{Abstract}

The composite preparation of plans is done in a coking plant to standardize the quality of mixtures. A recurring problem is the distribution of these coals in the constant feedweighter to be mixed. The distribution, in terms of these coals is done manually, taking into consideration only the knowledge of the operator. The proposal is to present a linear programming mathematical modeling to solve the problem of distribution of coals in constant feedweighter, as restrictions on the use of constant feedweighter within the optimum range, the mixture composition and standardization of belt conveyors, to reduce burden on the lines, with the purpose of running a coal blending plan. Operations research methods were used using the Simplex algorithm. Keywords: Linear programming; Operational research; Coal blending; Simplex.

1 Engenharia de Computação, Bacharel, Especialista em desenvolvimento de Automação e Instrumentação, Gerencia Geral de Tecnologia da Informação e Automação de Processos, ArcelorMittal Tubarão, Serra, Espirito Santo, Brasil.

2 Engenharia de Computação, Mestre, Especialista em desenvolvimento de Automação e Instrumentação, Gerencia Geral de Tecnologia da Informação e Automação de Processos, ArcelorMittal Tubarão, Serra, Espirito Santo, Brasil.

3 Engenharia de Software, Especialista, Especialista em desenvolvimento de Automação e Instrumentação, Gerencia Geral de Tecnologia da Informação e Automação de Processos, ArcelorMittal Tubarão, Serra, Espirito Santo, Brasil. 


\section{INTRODUÇÃO}

A produção de coque na ArcelorMittal Tubarão é realizada em 3 baterias, cada uma contendo 49 fornos com capacidade individual de 29 toneladas de carvão mineral seco, de diversas características e procedências.

O processo de coqueificação é demorado e exige cuidados para preservar a vida útil das baterias de fornos, a qualidade e o custo reduzidos do coque produzido e 0 volume do gás combustível gerado neste processo. Para obtenção de um produto (coque) mais adequado ao processo de produção do ferro-gusa líquido nos altosfornos, faz-se necessário uma mistura de carvões de forma adequada.

Observa-se que em uma usina integrada a mistura de carvões influencia em torno de $40 \%$ no custo de produção do ferro-gusa. Esta mistura é enviada para as baterias através de correias transportadoras e armazenada em duas torres de carregamento. Para a realização desta mistura é necessário que os silos utilizados sejam equipados com balanças dosadoras. Na ArcelorMittal Tubarão são utilizadas 12 dosadoras dispostas em 3 linhas de 4 dosadoras como mostra a figura (Figura1):

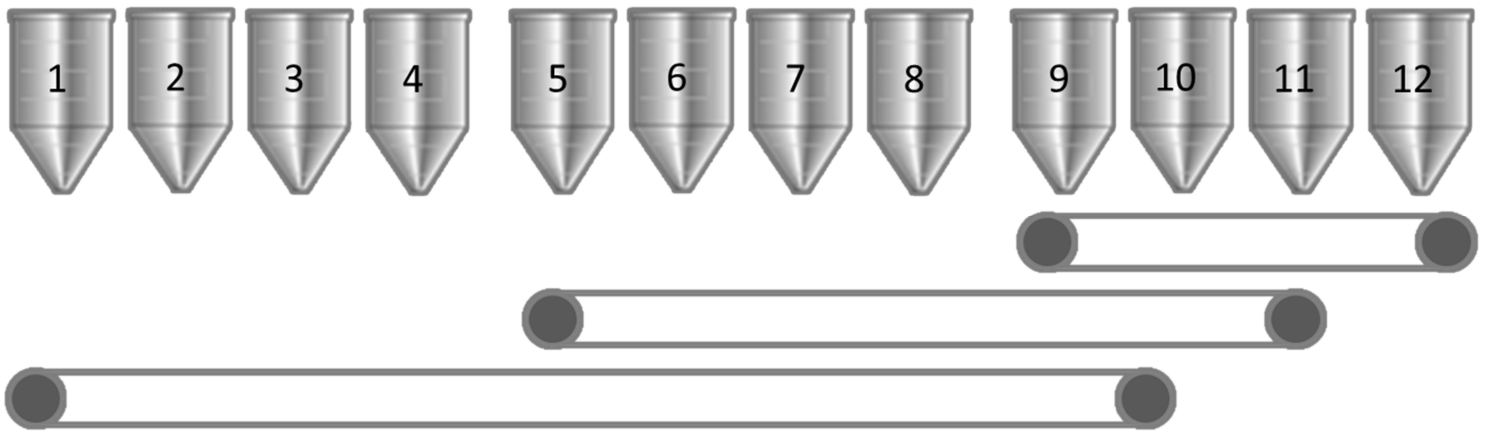

Figura 1. Disposição das dosadoras

Ao final de cada uma das 3 linhas possui um britador para ajustes granulométricos na mistura.

Para uniformizar a qualidade da mistura, é elaborado um plano de mistura, que consiste em uma receita de carvões e qual percentual de cada carvão será misturado.

\subsection{Arquitetura de Automação Existente}

Os sistemas de automação e controle industrial legados na empresa são formados por computadores isolados e sistemas operacionais e redes proprietárias. Alguns nomes utilizados para referenciar estes sistemas que de uma forma geral podem ser divididos em diferentes tipos de software e hardware:

- Sistemas SCADA (Supervisory Control and Data Aquisition) ou DCS (Distribution Control Systems);

- Interfaces Homem-Máquina (IHM);

- Controlador Lógico Programável (PLC-Programmable Logical Controller);

- Sistemas de otimização de processos e modelos matemáticos.

A figura 2 nos dá uma visão dos diferentes níveis hierárquicos de automação. Esta ilustração foi utilizada pela comunidade global de Automação da ArcelorMittal como referência para as fronteiras de atuação de cada nível. 


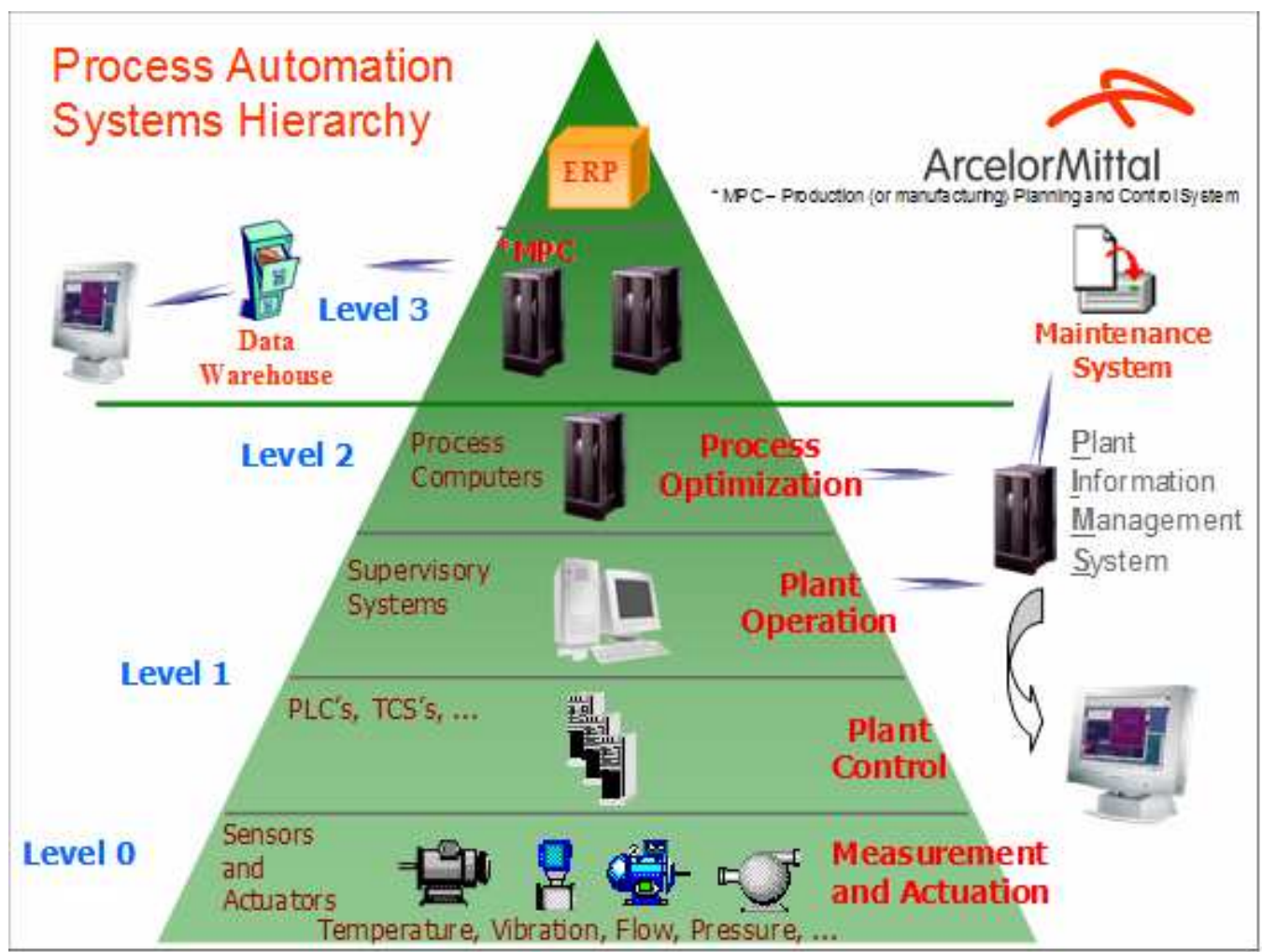

Figura 2. Arquitetura de sistemas de automação

$\mathrm{Na}$ área da Coqueria, o sistema de automação que suporta o processo de preparação de carvão, área foco deste trabalho, é o seguinte:

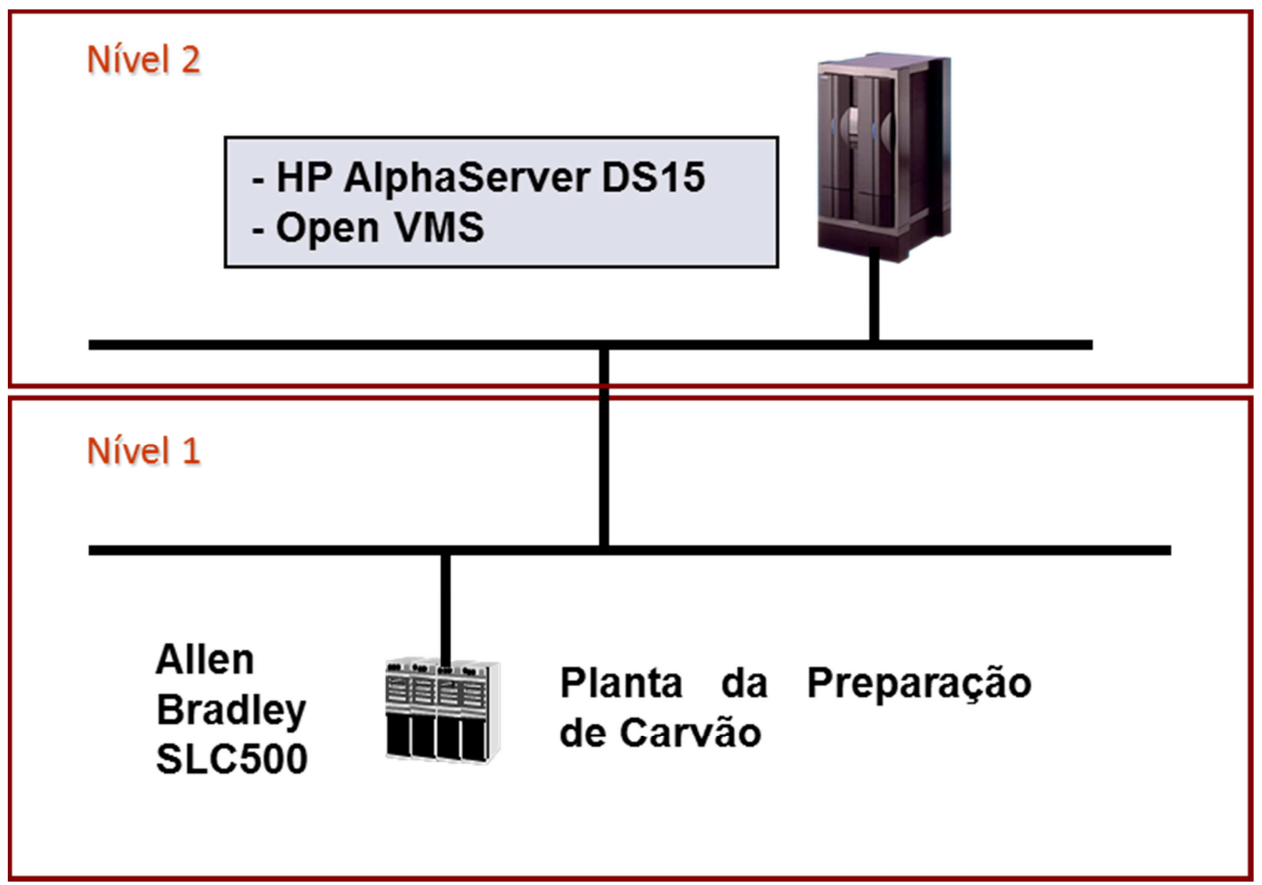

Figura 3. Arquitetura do sistema de automação da preparação de carvão

As balanças dosadoras estão ligadas ao PLC Allen Bradley SLC500 que disponibiliza para os sistemas de otimização os dados de vazão instantânea, integração e set point da vazão instantânea. 


\subsection{Caracterização do Problema}

Neste artigo ataca-se um item especifico que influencia a qualidade da mistura. De fato, a qualidade da mistura realizada pode ser influenciada por diferentes fatores, tais como a má qualidade do material usado, armazenamento inadequado destes materiais ou execução inadequada da mistura objetivada. E é sobre este último item que este artigo tenta contribuir.

O plano de mistura elaborado possui de 5 a 9 carvões, definidos pelas seguintes características:

- qualidade final desejada;

- custo;

- disponibilidade de carvões;

- manutenção das linhas de produção.

Atualmente, a distribuição, no plano, destes carvões nas dosadoras é feita de forma manual, levando em consideração apenas o conhecimento do operador, o que pode proporcionar a utilização não otimizada dos recursos disponíveis.

Um item específico que exemplifica a utilização não otimizada dos recursos está associado ao uso das balanças dosadoras. Estes equipamentos são especificados para trabalhar com precisão quando utilizados numa faixa de operação entre 20 a $80 \%$ do seu valor nominal. Medições fora desta faixa não são garantidas. Ou seja, dosadoras trabalhando fora da faixa ótima, podem influenciar diretamente na ocorrência de desvios na qualidade da mistura.

A Tabela 1 exibe as características de cada dosadora, com sua tonelagem horária máxima e sua faixa ótima de operação.

Tabela 1. Limites úmido das balanças das dosadoras

\begin{tabular}{lccc}
\hline Dosadora & $\begin{array}{c}\text { Tonelagem/horária } \\
\text { Máxima }\end{array}$ & $\begin{array}{c}\text { Limite } \\
\text { Inferior } \\
(\mathbf{2 0} \%)\end{array}$ & $\begin{array}{c}\text { Limite } \\
\text { Superior } \\
(\mathbf{8 0} \%)\end{array}$ \\
\hline 1 & 200 & 40 & 160 \\
\hline 2 & 150 & 30 & 120 \\
\hline 3 & 150 & 30 & 120 \\
\hline 4 & 150 & 30 & 120 \\
\hline 5 & 150 & 30 & 120 \\
\hline 6 & 150 & 30 & 120 \\
\hline 7 & 150 & 30 & 120 \\
\hline 8 & 150 & 30 & 120 \\
\hline 10 & 200 & 40 & 160 \\
\hline 11 & 150 & 30 & 120 \\
\hline 12 & 150 & 30 & 120 \\
\hline
\end{tabular}

Procurou-se então criar uma solução que permitisse orientar aos operadores qual a melhor distribuição dos materiais nas balanças dosadoras que garanta que as mesmas serão utilizadas dentro de sua faixa ótima de operação. Para isto, foi proposto o uso de um algoritmo de otimização conforme descrito nas seções seguintes. 


\section{MATERIAIS E MÉTODOS}

Para solução do problema apresentado na seção 1.1 optou-se pela utilização do algoritmo Simplex, dado que o modelo apresentado a seguir, na seção 2.1, apresenta características de sistemas lineares de otimização.

\subsection{Modelo Teórico}

O modelo teórico foi desenvolvido desacoplado da arquitetura de automação mostrado na seção 1.1 para levantar os possíveis problemas e validação do modelo. O foco inicial foi resolver o problema da alocação de carvões em dosadoras que se resume em alocar os carvões disponíveis nas 12 dosadoras de forma a otimizar a mistura. No entanto, este problema caracterizou-se complexo e dependente de outras variáveis, como alocação dos materiais no pátio no momento do recebimento, que deve ser previamente planejado para a recuperação de forma otimizada para as dosadoras, além da disponibilidade de uso das dosadoras. Decidiu-se então abordar este problema em outra ocasião. Assim, como premissa do modelo desenvolvido, os carvões já estarão previamente alocados nas dosadoras desejadas, cabendo então ao modelo distribuir os percentuais em cada dosadora. Consequentemente, a proposta deste trabalho é apresentar uma modelagem matemática e sua solução ótima, para o problema da distribuição dos carvões nas 12 dosadoras. As restrições impostas a este problema são:

- Garantia de utilização das dosadoras dentro da faixa ótima de operação;

- Garantia de composição da mistura segundo a receita;

- Utilização uniforme das correias transportadoras, para reduzir sobrecarga das linhas e paradas para sua manutenção.

Com base no problema proposto, o modelo matemático desenvolvido foi:

A função objetivo é expressa pela equação (Equação 1):

Em que,

$$
\max C=\sum_{j=1}^{n} x_{i j}
$$

C - percentual do plano distribuído nas dosadoras

$\boldsymbol{n}$ - quantidade de dosadoras disponíveis

$\boldsymbol{x}_{\boldsymbol{i} \boldsymbol{j}}$ - carvão 'i' alocado na dosadora 'j'

As restrições impostas ao modelo foram:

I. - restrições de uso da faixa ótima das dosadoras (Equação 2 e Equação 3)

$$
\begin{aligned}
& \left(\frac{V Z_{S E C A}}{100}\right) * x_{i j}>\left(L I_{j}\right), \text { para } j=1,2,3,4,5,6,7,8,9,10,11,12 \\
& \left(\frac{V Z_{S E C A}}{100}\right) * x_{i j}<\left(L S_{j}\right), \text { para } j=1,2,3,4,5,6,7,8,9,10,11,12
\end{aligned}
$$

Em que,

$V Z_{S E C A}$ - Vazão seca desejada para a mistura

$\boldsymbol{L I}_{\boldsymbol{j}}$ - Limite inferior úmido da balança 'j', valor descrito na tabela 1;

$\boldsymbol{L} \boldsymbol{S}_{\boldsymbol{j}}$ - Limite superior úmido da balança 'j', valor descrito na tabela 1; 
II. - restrições de balanceamento das linhas (Equação 4 a Equação 9)

$$
\begin{aligned}
& \sum_{j=1}^{4} x_{i j}>33.3(4) \\
& \sum_{j=1}^{4} x_{i j}<33.4(5) \\
& \sum_{j=5}^{8} x_{i j}>33.3 \\
& \sum_{j=5}^{8} x_{i j}<33.4 \\
& \sum_{j=9}^{12} x_{i j}>33.4 \\
& \sum_{j=9}^{12} x_{i j}<33.4
\end{aligned}
$$

III. Restrições da receita da mistura (Equação 10)

Em que,

$$
\sum_{j=1}^{12} x_{i j}=P L_{i}, \text { para } \mathrm{i}=\text { dosadoras que contém o carvão 'i' (10) }
$$

$$
\boldsymbol{P} \boldsymbol{L}_{\boldsymbol{i}} \text { - Valor percentual associado ao carvão 'i' no plano de mistura. }
$$

\subsection{Modelo Prático}

O modelo prático foi desenvolvido seguindo as diretrizes da arquitetura de automação mostrado na seção 1.1 .

O software foi desenvolvido em linguagem de programação Fortran77, sendo executado no hardware:

- HP Alpha Server DS15;

- 2.0GB (RAM);

- OpenVMS 7.3 (Operation System);

\section{RESULTADOS E DISCUSSÃO}

Para validação do modelo prático mostrado, foram utilizados planos de misturas reais utilizados na empresa. Para cada plano criou-se um cenário com os carvões utilizados, parâmetros do processo e limites impostos à vazão das balanças.

Os parâmetros a serem instanciados a cada plano são:

$\mathrm{n}$ - quantidade de dosadoras disponíveis

$\mathbf{V Z}_{\mathrm{SECA}}$ - Vazão seca desejada para a mistura

$\mathrm{LI}_{\mathrm{j}}$ - Percentual mínimo aceitável da vazão máxima da dosadora (Tabela 1)

$L S_{j}$ - Percentual máximo aceitável da vazão máxima da dosadora (Tabela 1) 


\subsection{Cenário 1 - Plano de Mistura A}

Os parâmetros utilizados para o cenário 1 foram:

$\mathrm{n}=12$ dosadoras

$\mathbf{V Z}_{\text {SECA }}=650 \mathrm{t} / \mathrm{h}$

$\mathrm{LI}_{\mathrm{j}}=20 \%$ da vazão máxima da dosadora 'j'

$\mathrm{LS}_{\mathrm{j}}=80 \%$ da vazão máxima da dosadora 'j'

Segue o resultado do cenário 1 :

Tabela 2. Plano de mistura $A$

\begin{tabular}{lccc}
\hline \multicolumn{1}{c}{ Carvão } & Porcentagem & Silo $\mathbf{1}$ & Silo 2 \\
\hline PD002TB & 15 & 1 & 6 \\
\hline PZ032TM & 25 & 3 & 10 \\
\hline Bl156TA & 10 & 4 & 7 \\
\hline ON045TM & 15 & 8 & 9 \\
\hline LS013TS & 5 & 12 & - \\
\hline GN084TM & 15 & 2 & 5 \\
\hline TO012TM & 15 & 11 & - \\
\hline
\end{tabular}

Tabela 3. Distribuição antes e depois do modelo

\begin{tabular}{cccccc}
\hline Dosadora & Carvão & $\begin{array}{c}\text { Operador } \\
(\mathbf{\%})\end{array}$ & $\begin{array}{c}\text { Operador } \\
(\mathbf{t} / \mathbf{h})\end{array}$ & $\begin{array}{c}\text { Modelo } \\
(\%)\end{array}$ & $\begin{array}{c}\text { Modelo } \\
(\mathbf{t} / \mathbf{h})\end{array}$ \\
\hline 1 & PD002TB & 7,5 & 52 & 6,2 & 44 \\
\hline 2 & GN084TM & 7,5 & 53 & 4,8 & 34 \\
\hline 3 & PZ032TM & 12,5 & 87 & 17,8 & 129 \\
\hline 4 & Bl156TA & 5,0 & 35 & 4,6 & 32 \\
\hline TOTAL L1 & & $\mathbf{3 2 , 5}$ & $\mathbf{2 2 7}$ & $\mathbf{3 3 , 4}$ & $\mathbf{2 3 9}$ \\
\hline 5 & GN084TM & 7,5 & 52 & 10,2 & 72 \\
\hline 6 & PD002TB & 7,5 & 52 & 8,8 & 62 \\
\hline 7 & Bl156TA & 5,0 & 35 & 5,4 & 37 \\
\hline 8 & ON045TM & 7,5 & 52 & 8,8 & 62 \\
\hline TOTAL L2 & & $\mathbf{2 7 , 5}$ & $\mathbf{1 9 3}$ & $\mathbf{3 3 , 2}$ & $\mathbf{2 3 3}$ \\
\hline 9 & ON045TM & 7,5 & 52 & 6,2 & 44 \\
\hline 10 & PZ032TM & 12,5 & 88 & 7,2 & 52 \\
\hline 11 & TO012TM & 15,0 & 105 & 15,0 & 105 \\
\hline 12 & LS013TS & 5,0 & 35 & 5,0 & 35 \\
\hline TOTAL L3 & & $\mathbf{4 0 , 0}$ & $\mathbf{2 8 0}$ & $\mathbf{3 3 , 4}$ & $\mathbf{2 3 6}$ \\
\hline
\end{tabular}

Para este cenário o modelo encontrou solução ótima, obtendo uma distribuição mais uniforme das linhas, com as restrições de vazão atendidas e as dosadoras trabalhando em uma faixa ótima. Observando a coluna de tonelagem horaria gerada pelo operador, é fácil ver que a linha 3 está sobrecarregada. Apesar da linha 2 passar a operar em uma velocidade maior, faz com que as outras linhas trabalhem de forma mais uniforme.

\subsection{Cenário 2 - Plano de Mistura B}

Os parâmetros utilizados para o cenário 2 foram:

$\mathrm{n}=12$ dosadoras

$\mathbf{V Z}_{\text {SECA }}=650 \mathrm{t} / \mathrm{h}$

$\mathrm{LI}_{\mathrm{j}}=20 \%$ da vazão máxima da dosadora 'j'

$L S_{j}=80 \%$ da vazão máxima da dosadora 'j' 
Segue o resultado do cenário 2 :

Tabela 4. Plano de mistura $B$

\begin{tabular}{lccc}
\hline Carvão & Porcentagem & Silo 1 & Silo 2 \\
\hline MA004TA & 13,5 & 6 & 3 \\
\hline ON018TM & 21,7 & 2 & 9 \\
\hline PN080TB & 13,0 & 1 & 5 \\
\hline BE007TM & 15,8 & 8 & - \\
\hline RK001TM & 13,0 & 11 & 4 \\
\hline VR030TS & 6,0 & 10 & - \\
\hline PZ005TM & 17,0 & 12 & 7 \\
\hline
\end{tabular}

Tabela 5. Distribuição antes e depois do modelo

\begin{tabular}{cccccc}
\hline Dosadora & Carvão & $\begin{array}{c}\text { Operador } \\
(\%)\end{array}$ & $\begin{array}{c}\text { Operador } \\
(\mathbf{t} / \mathbf{h})\end{array}$ & $\begin{array}{c}\text { Modelo } \\
(\%)\end{array}$ & $\begin{array}{c}\text { Modelo } \\
(\mathbf{t} / \mathbf{h})\end{array}$ \\
\hline 1 & PN080TB & 6,5 & 46 & 8,38 & 60 \\
\hline 2 & ON018TM & 10,9 & 77 & 15,17 & 107 \\
\hline 3 & MA004TA & 10 & 72 & 5,23 & 38 \\
\hline 4 & RK001TM & 6,5 & 45 & 4,62 & 32 \\
\hline TOTAL L1 & & $\mathbf{3 3 , 9}$ & $\mathbf{2 4 0}$ & $\mathbf{3 3 , 4}$ & $\mathbf{2 3 7}$ \\
\hline 5 & PN080TB & 6,5 & 46 & 4,62 & 32 \\
\hline 6 & MA004TA & 3,5 & 23 & 8,27 & 59 \\
\hline 7 & PZ005TM & 8,5 & 58 & 4,62 & 31 \\
\hline 8 & BE007TM & 15,8 & 111 & 15,8 & 111 \\
\hline TOTAL L2 & & $\mathbf{3 4 , 3}$ & $\mathbf{2 4 0}$ & $\mathbf{3 3 , 3}$ & $\mathbf{2 3 3}$ \\
\hline 9 & ON018TM & 10,8 & 76 & 6,53 & 46 \\
\hline 10 & VR030TS & 6,0 & 44 & 6,0 & 44 \\
\hline 11 & RK001TM & 6,5 & 44 & 8,38 & 56 \\
\hline 12 & PZ005TM & 8,5 & 59 & 12,38 & 86 \\
\hline TOTAL L3 & & $\mathbf{3 1 , 8}$ & $\mathbf{2 2 3}$ & $\mathbf{3 3 , 3}$ & $\mathbf{2 3 2}$ \\
\hline
\end{tabular}

Para este cenário o modelo encontrou solução ótima, obtendo uma distribuição mais uniforme das linhas, com as restrições de vazão atendidas e as dosadoras trabalhando em uma faixa ótima. Com base na Tabela 5 , pode-se ver que a distribuição feita pelo operador é relativamente bem balanceada, não sobrecarregando muito uma linha em detrimento da outra. Entretanto, ao verificar a dosadora 6 o operador não distribuiu de uma forma que a tonelagem horaria fosse maior que o mínimo desejado.

\subsection{Cenário 3 - Plano de Mistura C}

Os parâmetros utilizados para o cenário 3 foram:

$\mathrm{n}=12$ dosadoras

$\mathbf{V Z}_{\text {SECA }}=650 \mathrm{t} / \mathrm{h}$

$\mathrm{LI}_{\mathrm{j}}=20 \%$ da vazão máxima da dosadora 'j'

$\mathrm{LS}_{\mathrm{j}}=80 \%$ da vazão máxima da dosadora 'j'

Segue o resultado do cenário 3 : 
Tabela 6. Plano de mistura C

\begin{tabular}{lccc}
\hline \multicolumn{1}{c}{ Carvão } & Porcentagem & Silo 1 & Silo 2 \\
\hline BH179TB & 5,0 & 6 & - \\
\hline PZ032TM & 20,0 & 3 & 10 \\
\hline Bl156TA & 10,0 & 4 & 7 \\
\hline ON045TM & 15,0 & 8 & 9 \\
\hline BT007TB & 10,0 & 1 & - \\
\hline GN084TM & 15,0 & 2 & 5 \\
\hline TO012TM & 20,0 & 11 & - \\
\hline
\end{tabular}

Para este cenário o modelo não encontrou solução ótima. É possível ver que para o carvão "TO012TM" e a Vazão seca utilizada como parâmetro, a restrição de uso da faixa ótima das dosadoras não é atendida, logo o modelo não encontra solução ótima.

\subsection{Discussão}

Com base nos resultados demonstrados é possível ver que o modelo obteve uma distribuição mais uniforme das linhas, com as restrições de vazão atendidas e as dosadoras trabalhando em uma faixa ótima. Em alguns casos a restrição mais importante, que é a utilização das dosadoras na faixa ótima, é alcançada no seu limiar inferior ou nem é alcançada, fazendo com que o modelo não encontre solução ótima. Nesses casos, duas iniciativas estão sendo tomadas, sendo uma delas o préprocessamento dos dados antes da utilização do modelo, informando o operador que alguma das restrições não está sendo previamente atendida. Outra iniciativa é o pós-processamento das informações do modelo, que deve ser feita pelo especialista responsável pela criação do plano de mistura.

\section{CONCLUSÃo}

O modelo proposto consiste num modelo de programação linear de simples resolução. Após a análise dos resultados foi possível ver que a solução do método foi bastante eficiente. Alguns ganhos percebidos pelos usuários são:

- Minimização dos erros de dosagem, melhor observância dos limites técnicos das faixas de dosagem das balanças;

- Equilíbrio de dosagem por linha, evitando desagaste acentuado da linha de britagem;

- Padronização das ações de envio da mistura, evitar intervenção do operador na distribuição dos carvões no silo.

A busca pela otimalidade muitas vezes não é factível por que os planos de mistura não são criados com as restrições impostas pelo modelo, logo são gerados planos que não são otimizáveis.

Vale ressaltar que apesar do foco deste trabalho ser atuar em uma etapa de planejamento da mistura, os valores de percentual de cada material no silo estão diretamente ligados ao processo de misturas, pois no momento da execução do plano desenvolvido, o sistema de otimização calcula os set-points das balanças garantindo com que o modelo seja aplicado na vazão instantânea das mesmas.

Como proposta de pesquisa futura tem-se a extensão do modelo e do método de solução para alocação dos carvões nas dosadoras, e até mesmo criação automática do plano, a partir de uma qualidade pré-definida. 


\section{Agradecimentos}

Os autores agradecem as contribuições feitas pela unidade técnica da coqueria, a equipe de automação da área da redução da ArcelorMittal Tubarão e o Professor Luciano Lessa Lorenzoni pelo treinamento de técnicas de otimização.

\section{BIBLIOGRAFIA}

1 Araujo, Luiz Antonio de. Manual da Siderurgia - Vol.1 - Produção, Editora Arte e Ciência, São Paulo, 2005

2 Lorenzoni, Luciano Lessa. Técnicas de otimização - 2015

3 Padrões de operação das balanças dosadoras da Preparação de carvão da Coqueria da ArcelorMittal Tubarão - 2015

4 Programa de certificação Operacional ArcelorMittal Tubarão - 2006 